\title{
Seamless Health Monitoring Using 5G NR for Internet of Medical Things
}

\author{
Lalita Mishra $^{1}$ (D) Vikash $^{1,2} \cdot$ Shirshu Varma ${ }^{1}$
}

Accepted: 29 June 2021 / Published online: 8 July 2021

(C) The Author(s), under exclusive licence to Springer Science+Business Media, LLC, part of Springer Nature 2021

\begin{abstract}
Nowadays, information and communication technology grows rapidly. The microelectronics and communication mediums also enhance their reachability of coverage and connectivity. $5 \mathrm{G}$ enhances the capacity of the network in terms of lowest communication latency, highest speed, enhanced throughput, minimum E2E delay, and minimizing the packet loss. In this paper, we discuss the working principle and key features of $5 \mathrm{G}$ communication technology along with the limitations of existing technologies. Further, we provide the taxonomy of the $5 \mathrm{G}$ network. Moreover, we provide a comparison of $5 \mathrm{G}$ and $4 \mathrm{G}$ LTE in terms of data privacy and security aspects. Further, we propose a four-layer architecture for ehealthcare system, which uses 5G NR (New Radio) architecture incorporating the control plane and user plane. We perform the simulation over the frequency range 1 and frequency range 2 and calculated the throughput and latency for distinct values of OFDM numerologies. Further, we provide a comparative analysis for $4 \mathrm{G}$ and $5 \mathrm{G}$ and deduce that $5 \mathrm{G}$ facilitates 10 times lower latency than $4 \mathrm{G}$, and $5 \mathrm{G}$ can accommodate a much higher number of devices than 4G. In this work, we discuss providing better healthcare facilities electronically using 5G NR. Moreover, the data sharing and diagnosing the disease become faster and easier by using $5 \mathrm{G} \mathrm{NR}$.
\end{abstract}

Keywords Internet of medical things · Healthcare - Wireless sensor networks · 4G LTE · $5 \mathrm{G} \mathrm{NR}$

Lalita Mishra

rsi2018502@iiita.ac.in

Vikash

vikashcsiiita@gmail.com

Shirshu Varma

shirshu@iiita.ac.in

1 Department of Information Technology, Indian Institute of Information Technology Allahabad, Prayagraj, India

2 Department of Computer Science \& Engineering and Information Technology, Jaypee Institute of Information Technology Noida, Uttar Pradesh, India 


\section{Introduction}

From early 20th century (the information age), informatics is always an essential part of medications, along with the storage and management of the vast amount of data. As the Internet and communication technology have grown, the usage of computers is started in several other sectors of healthcare, other than only the storage of data, for example, sharing of the patient report to the specialist for getting best advise, in several cases outside the hospital, or even the city, from the hospital side or by the patients themselves

The digitization of healthcare industry is started years ago; however, the anticipated impact of the Internet on healthcare is not in the picture yet. The potential factors include the improvement in patient engagement, connected eCare, irresistant patient monitoring, and assisted lives for chronically effected and elderly people. This type of healthcare is beneficial at times when people are not that fit to even visit the hospital and they need e-prescription (prescribing medication on call or email by the doctor) and emedicine (home delivery of the medicine by the pharmaceutical company). In the present scenario of the entire world is consisting of people with increasing age. As per a status report published by WHO [1], the total world's population over 65 years of age was 702.9 million in 2019 and will be projected to approximately 1549 million by the year 2050, which shows the $120 \%$ augmentation. Similarly, if we talk about the population of people aging 80 years and above, it was about 53.9 million in 2019 and will reach up to 109.1 million by the year 2050, and showing a $102.6 \%$ overall increment. The global population of people suffering from dementia is around 50 million in the year 2019 and anticipated to reach up to 150 million by the year 2050, and among all these patients, approximately $80 \%$ belong to the age group of 75-95 years. Out of all these patients, over $70 \%$ will be dependent on the caregivers for their daily routine tasks. However, there is no possible alternative to reverse the cognitive limitations of the patient population.

The healthcare industry is exponentially growing and expanding marketplace, along with a huge increment in the number of applications, which will be beneficial by using the Internet like network. The data generated by this industry is also of various data types, sizes, and formats, which results in placing complex demands from the network itself, concerning network bandwidth, latency, data rate, and various other factors. The ehealthcare service begins with the sensor equipment deployed at different places of the healthcare centers which will further use the networking technologies to connect to the network, such as Wi-Fi, Bluetooth, and other related technologies. With the advancement of ehealthcare, there should be the advancements in connecting technologies, because the advancements require a huge number of devices and sensing types of equipment to run the sensing applications simultaneously in large ehealthcare centers, and this will ignite the rise in mMTC. The next in series is cMTC/ URLLC [2], which is highly useful in robotic remote surgeries over tactile Internet.

For coping up with these connectivity concerns, 5G, the cellular wireless technology with the fifth generation advancements, comes into the picture. $5 \mathrm{G}$ technology is having the potential to overture the power of massive connection along with very high speed, which results in on-time delivery of ehealthcare requirements, where a delay of even nanoseconds can make the situation worse. $5 \mathrm{G}$ is very much useful for the IoMT because it will take the new medical innovations (for example virtual reality, augmented reality, remote patient monitoring, remote healthcare learning, artificial intelligence, and many related technologies) into reality. Further, 5G is an essential technology in the field of medical IoT, as to access realtime data and make trice decisions out of it. The healthcare system demands the 
diagnosis and report generation should be done with high speed. Further, the file and image transmission required by ehealth system is bulk in size and it will take less transmission time if we use $5 \mathrm{G}$ transmission, because $5 \mathrm{G}$ provides ultra-low latency and better computation power, which will result in efficient communication and a rapid \& true diagnosis.

As the world move towards achieving quality of life for human beings, 5G plays an important role to achieve this goal. In this direction, [3-5] reported that approximately $16 \%$ of the worlds population will be in their elderly ages by the year 2050. This will cause rapid age-related disorders like neurodegenerative diseases and several types of sleep disorders. IoMT provides continuous, ubiquitous, and customized medical assistance to the customer and enhance the quality of life for the individual with reduced cost.

Although e-health and m-health had been identified as admissible and challenging objectives since the early phases of standardization in 2015, the enhanced communication system is needed for such kind of applications and investigated gingerly [4]. In this paper, we are trying to fill these gaps, especially in the identification of problems in ehealthcare which can get the maximum benefit of the usage of $5 \mathrm{G}$, describing the specific needs and requirements of $5 \mathrm{G}$, and will contemplate the impact of $5 \mathrm{G}$ on the Internet of Medical Things, that will promote the usage of $5 \mathrm{G}$ for the advancement of healthcare services, like connected ambulance and healthcare applications, like robotic surgery and remote surgery.

Further, the ehealthcare workers (whether the doctors or related paramedical staff supporting the doctor to provide the ehealthcare services) should have the medical knowledge as well as of scientific knowledge (usage of computers, information sharing, media sharing, saving \& retrieving data to and from the cloud storage). The healthcare literacy covers all these aspects and every ehealthcare worker must be proficient in all aspects.

Table 1 provides the list of abbreviations with the terms associated and the standard values of that term, as per applicability.

\subsection{Working Principle of 5G}

5G network is a digital cellular wireless network, in which the service area, is divided into tiny geographical locations, termed as cells. Fig. 1 shows the areal representation of two different types of $5 \mathrm{G}$ networks. The circular area represented by red color (bigger circle) is showing the range of $5 \mathrm{G} 600 \mathrm{MHz}$, covering the area of hundreds of square miles, and the circular area represented by a green color (smaller circle) is showing the range of $5 \mathrm{G}$ mmWaves, covering the area less than one square mile. In reference to Fig. 1, the smaller circle (unit area) represents the cell.

$5 \mathrm{G}$ works on the principle of cell cites, like other telecommunication technologies. These cells divide the designated territory into different sectors, and then send the data packets in encoded format of radio waves. $5 \mathrm{G}$ provides $30 \%$ enhanced speed with the same airwaves, used by $4 \mathrm{G}$. This enhancement in speed is because of the design of $5 \mathrm{G}$ channels, which are much larger (up to $100 \mathrm{MHz}$ ) than that of $4 \mathrm{G}(20 \mathrm{MHz})$.

$5 \mathrm{G}$ uses AMF (for receiving all the information related to connections and sessions from the UEs) and UPF (core component of 3GPP \& 5G infrastructure and is responsible to provide the information about interconnection points between the data network and mobile infrastructure) functions for connecting the NR to new gNBs. Thus, it performs the encapsulation and decapsulation mechanism on the GPRS tunneling protocol on the user plane.

The small cell works as the majority component of the $5 \mathrm{G}$ network, specifically for new mmWave frequencies using very short connection and communication range. Thus, 
Table 1 List of abbreviations with standard values (where applicable)

\begin{tabular}{|c|c|c|}
\hline Abbreviation & Term & Standard value \\
\hline LTE & Long-term evolution & \\
\hline NR & New radio & \\
\hline 3GPP & 3G Public-private & \\
\hline mMTC & Massive-machine type communication & \\
\hline MIMO & Multiple inputs multiple outputs & \\
\hline MEC & Multi-access edge computing & \\
\hline BAN & Body area network & \\
\hline $\mathrm{OCC}$ & Optical camera communication & \\
\hline cMTC & Critical machine type communication & \\
\hline URLLC & Ultra reliability and low latency communication & \\
\hline IoMT & Internet of medical things & \\
\hline RAN & Radio access network & \\
\hline gNB & Generation node base & \\
\hline UE & User equipment & - \\
\hline NVF & Network function Virtualisation & \\
\hline AMF & Access and mobility management function & \\
\hline UPF & User plane function & \\
\hline $\mathrm{Uu}$ & Interface between 5G-UE and 5G-RAN & \\
\hline $\mathrm{CA}$ & Carrier aggregation & \\
\hline OFDM & Orthogonal frequency division multiplexing & \\
\hline$\mu$ & OFDM numerology & $0-4$, we used $0-2$ \\
\hline FR1 & Frequency range 1 & $450-6000 \mathrm{MHz}$ \\
\hline FR2 & Frequency range 2 & $24,250-52,600 \mathrm{MHz}$ \\
\hline
\end{tabular}

for providing the continuous and undisturbed connection, the small cells are distributed in the form of clusters depending on the user's connection requirements. The small cell system is the complement of macrocell networking, which is dedicated to providing wide area coverage.

The 5G macrocells are using MIMO, which enables several connections for transmission (sending \& receiving) of more data at a time. The MIMO antennas, when using a huge number of antenna elements, are referred to as massive MIMO. It benefits the user by providing the capability of connecting more people to the network simultaneously and managing high throughput.

The core network is responsible for managing all the voice \& data exchange, internet connections, and works as the data and mobile exchange network.

\subsection{Challenges in Current (Physical) Healthcare System}

This section deals with the challenges present in physical healthcare system and how can be solved by using ehealthcare system [3]. Some major challenges can be addressed as: 


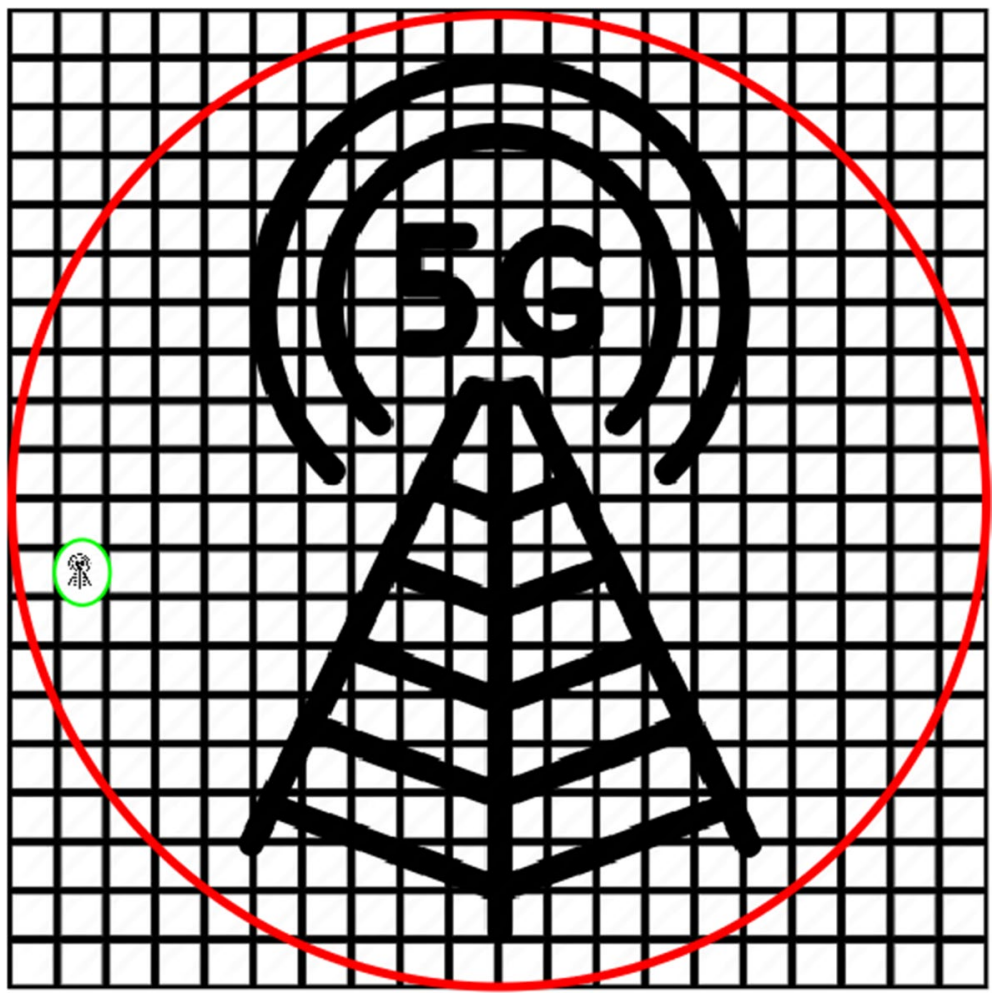

Fig. 1 Areal representation of 5G $600 \mathrm{MHz} \& 5 \mathrm{G}$ mm waves

\subsubsection{Record Maintenance}

The patient record maintenance is a tedious task in case of offline maintenance. This problem can be solved by opting Electronic Health Records (EHR). EHR is a digital repository for collection and maintenance of patient's record and prevent the unauthorized access by implementing secure exchange with authorized users only. EHR support interoperability by connecting the hospitals and patients through internet. By using EHR, one patient's data can be shared with several experts in all the manners, that are retrospective, concurrent, and prospective manners.

\subsubsection{Access to Healthcare Facilities}

In the present healthcare scenario, each healthcare facility cannot be provided to everybody, because of the nation boundary, patient is critical and has less time, long distance to travel, cost incurred in traveling and availing facilities. This challenge can be solved to a much extent through ehealthcare syatem, which provide the equal access to any and every type of healthcare facilities to everyone, through internet, without any boundaries of cities or nations. 


\subsubsection{Personalization in Medication System}

In the present healthcare scenario, the patients having same disease are prescribed with same medicine. However, there are three types of patients in terms of responding to medication. First, the patients responding for some medication, second are not responding for medication, and third are showing adverse effects for the same medication. Using ehealthcare system can help in changing the treatment plan, before the medication effects severely the patient. This is because, with ehealthcare the patient will not bother to go to the hospital, take the appointment, then meet with the doctor with the side-effect problem. The patient will send his the data to the doctor and get immediate changes.

\subsubsection{Limitation of Information Flow}

Wireless communication technologies, like, 3G, 4G, etc., are suitable for wellness monitoring applications and smart health monitoring devices. However, for a better ehealthcare system a heterogeneous technology is required which provides multiple frequency ranges along-with low latency and high data rate (for remote surgery), able to exchange data in more secure manner (prevent data theft). With the help of Tables 3 and 4, discussed in Sect. 6, we can say that $5 \mathrm{G}$ fulfills all these requirements of information flow.

\subsection{Our Contribution}

The main contributions of the paper are listed as follows:

- We propose a 5G architecture for ehealth care system along with the taxonomy of 5G, which is a unique step for ehealth care applications.

- We have also present necessary and important characteristics of 5G communication and provide a comparative view with other similar technology like LORA, SIgFox, etc.

- We have evaluated the performance of $4 \mathrm{G}$ and $5 \mathrm{G}$ over different matrices by creating a comparable environment in NetSIm. In this simulation, we have considered various physical and environmental aspects like frequency range, carrier aggregation, and noise.

The rest of the paper is organized in total 12 sections. Section 2 consists of literature survey. Section 3 summarizes the encountered limitations of using existing technologies in ehealthcare system. Section 4 describes the important features of 5G NR and Sect. 5 discuss about the technologies leveraged with 5G. Further, Sect. 6 illustrates the detailed taxonomy of 5G for IoT applications and Sect. 7 compares the 4G LTE and 5G NR on the basis of data privacy and security. Section 8 provides the system architecture of 5G NR for ehealthcare system and in Sect. 9 we discuss about specific IoMT applications and how 5G is useful for that particular application. Moreover, Sect. 10 provides the overall architecture of 5G NR with control plane and user plane. The results are described in Sect. 11 and section 12 deals with conclusion and future scope of our work. 


\section{Literature Review}

Soldani et al. [6], presented a 5G based mobile healthcare system incorporating ultra-reliability and low latency features of 5G. They mainly focus on wireless telesurgery (WTS) and wireless service robots (WSR). The WTS uses a mobile console and robotic platform for sharing its feedback in audio, video, and haptic format, whereas WSR is a companion robot performing the task of social caretaker in the form of a family member or professional personnel. Although, they have not considered the throughput, CA values and the two frequency ranges of 5G, FR1 and FR2, which are important aspects of 5G communication. Rehman et al. [7], used the concept of small cells to enhance medical QoS and medical QoE experience in terms of frequency efficiency, network traffic, and energy and cost reduction. They found how small cells affect the heterogeneous networks for streaming medical videos in the uplink direction, and the effect of packet loss in medical video streaming. They also introduce the concept of mobile small-cell network-enabled ambulance, which show the better simulation results in terms of throughput, delay, and packet loss, over the macro-cell based network enabled ambulance. However, they have also not considered the CA values and the two frequency ranges of 5G, FR1 and FR2. Yao et al. [8], present an artificial intelligence-based scenario of healthcare enabled with 5G communication network. They discuss the AI-based healthcare applications for solving network deployment and management issues in 5G along with two different architectures of physical and MAC layer processing. They proposed a spectrum access system (SAS) in 5G and get an accuracy of $20 \mathrm{MHz}$ for $3.5 \mathrm{GHz}$ shared band for 802.11ac and LTE signals. Ahad et al. [9], presented a 5G architecture for smart healthcare using device-to-device communication, network function virtualization, small cells, software-defined network, edge computing, and mmWaves. Further, they provide a review of various network layer possibilities (scheduling, congestion control techniques, and routing) in a combination of the usage of the Internet of things in the healthcare domain.

Nasimi et al. [10], provided a congestion control technique based on MEC, aiming for taking the real-time decisions for the selective buffering network traffic, for the enhancement in QoS. However, they never consider any other aspect except delay and packet loss in their paper. Cisotto et al. [4], identified the healthcare scenarios and talk about the need for a $5 \mathrm{G}$ network for the proper and efficient functioning of the healthcare system. Further, they used the concepts of network slicing, managing the heterogeneous network, and mobile edge computing for providing mhealth and ehealth solutions using $5 \mathrm{G}$ networks for ubiquitous medical care. Although, everything is discussed in theoretical aspects, no results are shown by the authors.

Chowdhury et al. [11], designed a monitoring system for monitoring the patient in home, hospital, outdoors, intensive care units, and ambulances. They used OCC for monitoring the patients. The monitored data from wearable sensors/patches are received by the OCC system, which is connected to the $5 \mathrm{G}$ access networks. The system is able to monitor several patients at the same time and transfer the monitored information to the hospital server, medical server, doctors, mobile systems, and clouds for remote monitoring purposes.

Wang et al. [12], surveyed several wireless network optimization and design aspects including modeling \& estimation, channel measurement, network measurement, and optimization. However, they did not provide any results for their theoretical assumptions

Ahmed et al. [13], discussed the concept of hospitals of the future (HoF) and the wireless connectivity required for it. The $\mathrm{HoF}$ will connect the healthcare professionals, 
patients, medical devices, sensors, and computers, wirelessly, for gathering and analyzing the patient's health data. They proposed a hybrid flexible optical radio wireless network for providing high performance and efficient wireless connectivity. The network can be reconfigured dynamically for transmitting and receiving the optical or radio or both types of signals, as per the application need. Liu et al. [14], presented a survey regarding the latest advancements in the field of healthcare, such as monitoring devices, eHealth systems, robotics, AR/VR, and AI-based applications. The monitoring devices include in-body and on-body devices serving the purpose of monitoring the patients and are used in hospitals and homes.

Annapoorani et al. [15], presented a smart healthcare monitoring system (SHMS) for monitoring the health conditions of elderly people. They designed the system using a MEMS sensor, temperature sensor, respiratory sensor, and heartbeat sensor. If any abnormality is found in the sensed data, the information is sent to the doctors along with the family members automatically by using the mobile application. However, they discussed all the things with respect to sensed data, but they did not talk about communication delay, which is important in communicating sensed data. Dzogovic et al. [16], tested the deployment of network slicing mechanisms in terms of healthcare vertical enabled with $5 \mathrm{G}$ technology. They used the concept of 5G core along with the software defined networks. However, several SDN controllers are needed, having distinct features (capability and performance) for achieving the network slices in 5G.

In [17] Ioannou et al. proposed a distributed intelligent method for controlling the generation of device-to-device networks, using belief-desire intension (BDI) and extended belief-desire intension (BDIx) for satisfying the challenges occurred during device-to-device communication. However, they had not presented any analysis about by which factor proposed scenario help in improving existing system. Cui et al. [36], presented the concept of smart walls for the healthcare domain. The mmWave spectrum is used for providing enhanced throughput, low latency, and communication reliability along with broad coverage. The intelligent walls are able to manipulate signals for providing an interference-free and full-duplex communication link between the base station and the user. Although, they did not consider the throughput and delay aspects of wireless communication. Further, Chen et al. [18], proposed a cognitive system for emotion recognition, but, they did not metion how $5 \mathrm{G}$ is necessary for the system to work well using characteristic feature of wireless communication.

Hewa et al. [19], proposed a secure service architecture using elliptic curve QuVanstone based on MEC and blockchain for providing user authentication, real-time data integrity, and privacy among MEC, IoT, and cloud. Moreover, they also attached a storage offloading capability to blockchain for ensuring scalability in connected medical devices. They have evaluated the proposed architecture by the Hyperledger Fabric blockchain platform along with Raspberry Pi in a near real-time scenario. Ning et al. [20], constructed a health monitoring system based on game theory by dividing the network into Intra-WBANs and beyond-WBANs, in a costefficient manner. The cost is dependent on the age-of-information, medical criticality, and energy consumption by the IoMT devices. Although, they had not considered the communication delay, throughput, speed, etc., features of wireless communication.

Any of the paper did not considered the carrier aggregation (CA1 and CA2) values, and the frequency ranges (FR1 and FR2) of 5G communication technology. We have incorporated these aspects of $5 \mathrm{G}$ in this paper along with the communication delay, throughput, and latency values. 


\section{Features of 5G Communication}

In this section, we discuss some of the 5G NR features, which are useful in developing efficient ehealthcare system and maintaining the usage and storage of the patient's data in a secure manner. Some important features of $5 \mathrm{G}$ communication are as follows:

\subsection{Communication Speed}

Early use of ehealthcare incorporate both benefits and limitations of existing communication technologies, for example, the tele-echography platform which has carried into effect using LTE cellular link, providing with $10 \mathrm{Mbps}$ data rate, $60 \mathrm{~ms}$ of latency, and $8 \mathrm{~ms}$ of jitter. The fifth-generation cellular network provides a significant improvement over LTE cellular link over data rate, latency, reliability, and jitter. The communication speed attained by using $5 \mathrm{G}$ is starting from 10 to $25 \mathrm{Gbps}$, which helps for transmitting the patient's report to the doctor with minimum possible delay.

\subsection{Response Time}

It is the actual time, consumed by the system to come in execution state, which works on the principle, the faster the speed, the lesser the response time. 5G provides lesser response time than $4 \mathrm{G}$ and thus, it is a mile stone for real-time applications like ehealthcare, self-driving cars, missing person's video surveillance, and other time-sensitive applications.

\subsection{Connection Density}

The number of connected UE per unit area, provided with full penetration $5 \mathrm{G}$ networks. Moreover, density is the ability to support successful delivery of information for certain packet size within the time frame along with the space constraints. In near future, we are expecting to support up to 1 million user devices per 0.38 square miles in 5G [21] (most important for a better ehealth system) whereas 4G supported 2000 devices in per 0.38 square miles.

\subsection{Survival Time}

It is the maximum possible packet delay tolerable by the specific application to perform the designated operation properly. After that the receiving node sends the negative acknowledgment and pass the command for resend the packet. This property is useful in ehealth while transmitting the reports of the patient in emergency situation.

\subsection{Network Management and Operational Support System}

The communication operators are always trying to reduce the network overhead and thus, reduced operating expenses. The reduced network overhead will lead to better management of network and operational support system (OSS), which further will result 
in improved resilience, serviceability, consistency, analytical capability, reliability, operational efficiency, and availability of the network to build a better ehealth system.

\subsection{User Mobility Support}

All of us experienced the loss of connection while traveling from one place to another. 5G provides high-speed user mobility support, up to a hundred kilometer per hour. Thus, it has the ability to support all modes of communication, for example, from car to aircraft, the communication and connection will not be lost, no matter through what means you are traveling with. This feature of $5 \mathrm{G}$ is essential at the time of using an air ambulance. Some severe patients need to be shifted from one hospital to another (inside and outside the boundaries of the nation) for better treatment. If the ambulance is connected to the hospital server, then the hospital authorities are aware of the condition of the patient, as well as the time of arrival to the hospital, so that they can prepare the essential set up before the patient come to the hospital and start the treatment as soon as the patient arrive at the hospital.

\subsection{Universal Application Support}

5G inherited the features of its predecessor technologies along with facilitating the three unique aspects of wireless communications. First is, the fastest data delivery from $1 \mathrm{Gbps}$ to $50 \mathrm{Gbps}$ in some test environments. Nevertheless, the $5 \mathrm{G}$ is anticipated to provide the data transfer speed of $2.8 \mathrm{Gbps}$ in real-world scenarios, which is much higher in comparison with $4 \mathrm{G}$ (60 Mbps). Second is, it caters to the latency of one millisecond, which is much lower than the latency provided by $4 \mathrm{G}(50 \mathrm{~ms})$. The third aspect is, the connecting capacity of more devices simultaneously. Through $5 \mathrm{G}$, we can connect up to one million devices per kilometer square, which is much higher than that of 4G $\left(60,680\right.$ devices $\left./ \mathrm{km}^{2}\right)$. Thus, $5 \mathrm{G}$ can support various healthcare applications, like, connected ambulances, remote surgery, etc., because 5G uses the device-to-device, MIMO, mmWaves communication technologies with full duplex transmission capacity. The full duplex transmission provides a coverage of rural-to-urban, roadsides-to-railway stations, railway tracks-to-on air (air ambulace and drone) coverage to the ehealthcare facilities [3].

\subsection{Security}

The 5G makes the security of the network stronger than any of the existing communication technologies (for example, 3G, 4G, 4G LTE, 4G Pro, etc.) by including additional preservation at the edge of the network, network slicing, visualization of network functions, and mobile edge computer, all of which makes the network more secure. The Verizon telecommunication company encompasses a new 5G security tool in its security plan, which sequesters the mobile devices under roaming until and unless they are properly authenticated to be used in a particular network. This will help in secure data communication, as vague data transmission leads to data leaks. 


\subsection{Privacy}

The advancements in data mining results in the retrieval of user's privacy information in an easier way and the leakage of private information will drive grievous consequences. 5G cater to many vertical industries as well, thus, it must make sure that the user's and industrial privacy information is protected so that the network can be used without bothering about the information leakage. For enabling more privacy protection, 5G enables customized network (slicing and selection network) services for its user by sensing the type of services used by the user (for example, location and health related information). For heterogeneous type networks (using multiple access technologies), the privacy protection is provided depending upon the access network and technology they are using.

The security and privacy of data is very important in ehealth system to safeguard the patients from fraudulent claim of insurance and unauthorized use of their medical prescriptions.

\section{Leveraging Technologies by 5G}

The heterogeneous wireless network is consisting of several access networks, cells, frequency bands. All of these come together with overlapping coverage areas, which cause network planning and deployment, a challenging task for wireless operators. However, 5G comes with some important technological enhancements, that will implement IoT applications in real-time, more easily and efficiently. 5G preferably provide network virtualization, next level navigation system, network slicing, cloud services, self-organizing networks, mobile IoT, artificial intelligence, and eSIM technologies, which are discussed in brief as follows:

\subsection{Virtialization}

In service-based 5G core network architecture, all operations are performed by using functions and are performed outside the operating network, like, the cloud. This type of enabled virtualization requires convenient isolation controls to be incorporated in because, without it, the network is vulnerable to data leakage and virtualization-aware malware outbreaks.

Containerization, which provides OS-level virtualization, is a popular technique of virtualization at present. In this, the host operating system restrains the containers accessibility to the physical resources of the system, for example, the storage, CPU, and memory. Thus, a single container is unable to use all of the host system's physical resources, and thus, it reduces the influence of availability attacks concerning the platform.

SDN (Software defined networking) provides the network operators, a chance to virtualize the flow of the network, which results in the ingeniosity of hardware.

\subsection{Next Level Navigation System}

The high speed and low latency features of $5 \mathrm{G}$ enable it to use in the navigation system to the next level. 5G networks and communication technology can handle a huge amount of data and a higher number of connected devices simultaneously. This will help in making the navigation system faster, reliable, and hassle-free in real-time. It will provide improved efficiency and reliability in self-driving and connected vehicles. Better navigation can help 
in healthcare by showing the smallest route with lesser traffic to the hospital so that the connected ambulance can reach the hospital in minimum time frame.

\subsection{Network Slicing}

Network slicing assents the creation of several virtual networks on the top of a commonly shared physical infrastructure [22]. Further, network slicing is used to design a virtual architecture of network, which is working on the exact principle of NVF and SDN in fixed networks. Network slicing is an essential aspect of $5 \mathrm{G}$ networks, as in fifth generation network, one physical network is virtually isolate from several RANs.

According to GSMA Intelligence (a source of mobile operator data, it's insights and related forecasts), the number of $5 \mathrm{G}$ connections will be 1.2 billion, which will be $40 \%$ of the global population (estimated to 2.7 billion), by the year 2025 . They hypothesize about $5 \mathrm{G}$ network that, it will provide an actual opportunity to design a resilient network which accommodates the distinct requirements of particular industries and economy of it.

\subsection{Cloud Services}

Nowadays, everyone is using mobile applications, which are based on cloud services. 5G makes the ease of availability because of it's characteristics like low latency, higher speed, and service reliability. By these features, cloud computing provides smoother communications to mobile users. Cloud services using $5 \mathrm{G}$ will boost the performance of the applications. Moreover, 5G enables cloud service providers to approach mobile customers more reliably and easily. Access to a virtual machine using mobile phones is becoming more usual because of the greater computing power and machine-to-machine communication provided by $5 \mathrm{G}$.

\subsection{Self-Organizing Networks (SON)}

The self-organizing capabilities [23] of the networks maximize the performance of the cellular networks. The SON property is referring to the automation of mobile networks and minimized human interventions in wireless or cellular network management. SON enables the autonomous and intelligence acclimatization, reduction in operational and capital expenditure, and enhance the network performance in views of coverage, computation power, capacity, offered services, spectral efficiencies, and user experience provided by cellular networks.

Further, SON derives to self-configuration (automatizing the deployment phase of the network) self-optimization (adjusting the network parameters to optimize the performance of operating network), and self-healing (detecting, preventing, and correcting network failures) networks.

\subsection{Mobile Internet of Things}

Mobile Internet of things (MIoT) [24] can be defined as an IoT system that is enabled to move and reuse for different geographical locations. MIoT system can be used in various real-world application domains, for example, housing, education, entertainment, healthcare, etc. 
It is an estimate that the number of connected IoT devices will reach up to 3.2 billion by the year 2023. Further, MIoT provides a fast and efficient way of building new systems for a consumer using the MIoT system. The MIoT will help in the healthcare domain in managing the patients and healthcare workers by monitoring patient's current health status, documenting patient data, performing statistical computation on health data, and providing the connection to the hospital and other available health information systems. 5G technology supports the MIoT healthcare support at NR-Light band, which provides the lowest delay tolerance and lower complexity.

\section{5G Taxonomy}

Figure 2 shows the taxonomy of $5 \mathrm{G}$ for IoT applications. Figure 3 is showing a layer by layer description of the $5 \mathrm{G}$ scenario. First, we need to decide the objectives to be obtained, then the requirements for accomplishing the objective need to be figure out. The third layer is showing the approaches for the implementation of the system. The fourth layer is showing the communication technologies, and the fifth layer deals with the performance matrices. All five layers can be described as follows.

\subsection{Resource Optimization}

The main goal of optimizing the resources used in a network is to enhance the network lifetime, by minimizing the energy consumption by the UEs. In the case of IoT applications,

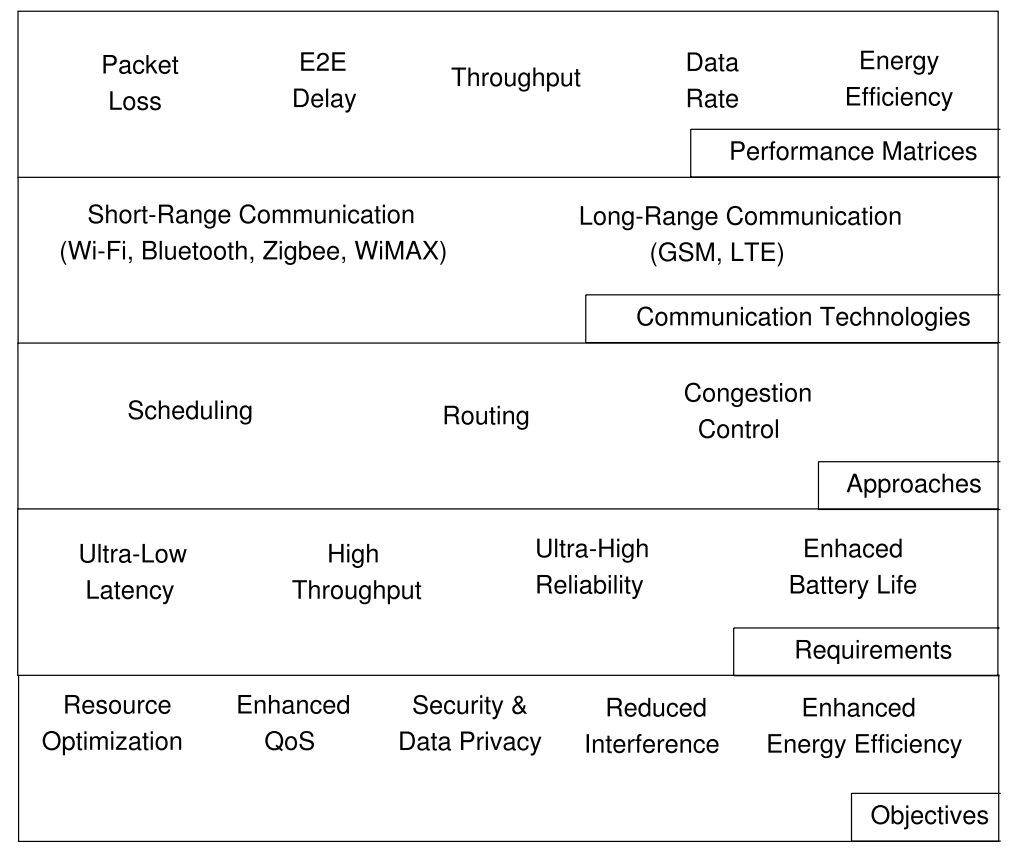

Fig. 2 Taxonomy of $5 \mathrm{G}$ 
Table 2 Requirements and availability (in 5G) of distinct features for eHealth system

\begin{tabular}{|c|c|c|}
\hline Features & Required value for ehealthcare system & Provided by $5 \mathrm{G}$ \\
\hline Bandwidth & $\begin{array}{l}\text { Nursing home }-10 \mathrm{Mbps} \text { Clinic/Large Physi- } \\
\text { cian Practice }(5-25 \text { physicians })-25 \mathrm{Mbps} \\
\text { Hospital-100 Mbps Academic/Large Medical } \\
\text { Center-1000 Mbps }\end{array}$ & $\begin{array}{l}20 \text { Gbps for downlink } \\
\text { and } 10 \text { Gbps for } \\
\text { uplink }\end{array}$ \\
\hline Data rate & $100 \mathrm{Mbps}[25]$ & $20 \mathrm{gbps}$ \\
\hline Communication latency & $1-10 \mathrm{~ms}[25]$ & Upto $1 \mathrm{~ms}$ \\
\hline
\end{tabular}

the network or system must not be drained out because of the extensive battery usage, because it can cause loss of a person's life, who is in the need of ehealthcare.

\subsection{Enhanced QoS}

The main measures that need to take into account for the calculation of QoS are jitter, latency, packet loss, bandwidth, etc. Further, we have already discussed that 5G provides low jitter, ultra-low latency, reduced packet loss, etc. Thus, the use of $5 \mathrm{G}$ networks is going to be a game-changer in IoT applications by providing enhanced QoS to the system.

\subsection{Security and Data Privacy}

The privacy of data is dealing with storage of data related to every patient in a confidential manner. As, if there is any breach in data privacy, some unauthorized person can use that data for claiming fraudulent medical insurance claims, generate illegal prescription and purchase the medicines on behalf of that prescription, illegally purchase some medical equipments, and sale it later on. For preventing such medico-legal frauds, the data must be stored in a secure and private manner.

\subsection{Reduced Interference}

The frequency reuse concept can be applied to the IoT applications for better resource utilization. Further, there is dense data traffic on the network in the eHealth scenario. Thus, the sharing of the efficient load should be improved, between the local access networks and the $5 \mathrm{G}$ macrocells. However, this advancement comes up with a limitation of co-channel interference caused by increased load and density on the network. Hence, we require efficient interference reduction schemes for the IoT applications to work efficiently and effectively.

\subsection{Enhanced Energy Efficiency}

The energy required for transmitting the data packet is dependent on the type of network used [26]. 5G communication consumes approx 90\% less energy than 4G. Energy consumption is an important aspect of communication, as the battery life of the mobile IoT devices is directly proportional to energy consumed by the IoT devices. 


\subsection{Ultra-Low Latency}

5G communication technology is facilitated with fast and real-time acknowledgment, which is a must in IoT applications by providing low latency while the data packet is traveling from one end to another on the network (for example, from patient to doctor or hospital server and vice versa). 5G provides an order of less than $1 \mathrm{~ms}$ of latency [27], for communicating the data packets, which is 4-5 times faster than $4 \mathrm{G}$ communication, and essential for time-sensitive real-time applications, like ehealthcare, automatic car, etc.

\subsection{High Throughput}

Throughput is the amount of data, that is transmitted through a channel. As we discussed, $5 \mathrm{G}$ provides much higher transmission speed, thus is providing higher throughput, which further results in better IoT applications related bulk data (files/reports/images) transmission and faster diagnosis and results.

\subsection{Ultra-High Reliability}

Reliability is a probabilistic measurement of delivering the data packets of the network layer successfully to the system entity within the given time constraint by the target service. It is also called as the degree of reliability. It must be high in an IoT applications.

\subsection{Scheduling}

The scheduling of resources is an important aspect of using the resources optimally in a 5G environment. Yan et al. [28] presented an intelligent resource scheduling strategy (iRSS) for RAN slicing in a $5 \mathrm{G}$ environment. The iRSS provides an idea for a collaborative learning framework including reinforcement learning and deep learning mechanisms for the allocation of resources. In [28], deep learning enables the large time scale resource allocation, and reinforcement learning is used for providing online resource scheduling to handle the small time scale network parameters, such as unexpected network states and inaccurate predictions. The iRSS is able to assign specific tasks for predicting an online decisionmaking modules in a flexible manner for assisting the RAN to make the right resource scheduling decision based on available historical data.

\subsection{Routing}

Khan et al. [29] presented a two-level clustering scheme for data dissemination in an efficient manner in a $5 \mathrm{G}$ environment for the vehicle to everything (V2X) communication. They use the fuzzy logic for the selection of level one cluster head (L1CH). The fuzzy logic uses the relative velocity factor (RVF), k-connectivity factor (KVF), and link reliability factor (LRF) for the selection of L1CH. Further, the 5G V2X is supported by two modes (mode 3 and mode 4) for $\mathrm{V} 2 \mathrm{~V}$ communication. The resource allocation is done by cellular networks in mode 3 , however, there is no need for cellular network coverage in mode 4. The vehicle selects the required radio resource autonomously by using the distributed 
scheduling scheme. The vehicles are connected to each other by mode $4 \mathrm{~V} 2 \mathrm{~V}$ communication, however, the exchange of data to and from the network is taking place with the help of a gateway vehicle enabled with cellular Uu interface.

\subsection{Congestion Control}

Najm et al. [30] presented a congestion control technique based on the decision tree approach using machine learning, which predicts the optimal enhancement in controlling the congestion in 5G IoT wireless environment. They have implemented the prediction model on a training dataset for determining the optimal parametric settings for the 5G scenario. The dataset is used to train the ML-based model and enable for predicting an optimal alternative for performance enhancement along with congestion control. The decision tree algorithm is used specifically for prediction and classification and provided better results with $92 \%$ precision and recall.

\subsection{Short-Range Communication}

The digital transformation of the healthcare system is in demand for exchanging the health related data. The communication technologies used for such data exchange in a short-range (up to $100 \mathrm{~m}$ ), are Bluetooth, Bluetooth low energy, Wi-Fi, Wi-Fi direct, near field communication (NFC), etc. Here, Wi-fi direct is used to transfer data among the devices, whereas BLE and Bluetooth are used for transferring the data among peripherals [31]. The shortrange communication technologies are specifically useful in the BANs [9].

\subsection{Long-Range Communication}

The long-range communication technologies are used for transportation of the health related data up to kilometers depending on the range of the applied technology (WiMAX $50 \mathrm{~km}$, LoRa $25 \mathrm{~km}$, LoRaWAN 15-45 km, SIGFOX 50 km, EC-GSM $100 \mathrm{~km}$, etc.). This data exchange is performed from the local server to the base station of the healthcare system. LoRaWAN supports the interoperability among many operators, while SIGFOX is using ultra-light band radio technology along with the full-star based network design for providing a highly versatile network for IoT applications with a very low power consumption [9].

\subsection{Packet Loss}

Packet loss is the average data packets that are lost during their transmission between the source station and the destination on the network. There should be minimum loss of packets on a network to work efficiently.

\subsection{E2E Delay}

End-to-end delay can be defined as the transmission time taken by the data packets for traveling from source end to destination end. The minimum is the E2E delay, the better is the network performance. 


\subsection{Data Rate}

It is defined as the acceptable rate of data delivery by the user for any specific service. Further, $5 \mathrm{G}$ has the target to improve the data rate by 10-100 times, in comparison with LTE. The ehealth system needs a data rate of $100 \mathrm{mbps}$ and $5 \mathrm{G}$ is able to provide it Table 2.

\section{Comparison of 5G with Existing Wireless Technologies}

Table 3 provides the comparison of $5 \mathrm{G}$ with existing wireless technologies. We compared the technology in terms of Latency (how much time taken to transmit), Distance (coverage area), Speed (how fast the data is transmitted), and Power (battery drainage), which are essential components in wireless transmission. Here, we include LPWAN technologies (licensed and unlicensed), classic Bluetooth, BLE (Bluetooth Low Energy), Zigbee, and WLAN, to compare with IoT. The unlicensed LPWAN covers Sigfox, LoRa, and Ingenu technologies, whereas licensed LPWAN covers LTE-M (Modifide LTE), NB-IoT (Narrow Band IoT), and EC-GSM-IoT (Extended Coverage GSM IoT).

\subsection{Comparison of 5G-NR with 4G-LTE}

Table 4 provides the comparison of 5G technology with the existing LTE. The comparison is done based on privacy and security aspects and methods to provide the same, in both the technologies. By referring to Table 4, we get ensured that $5 \mathrm{G}$ provides, better security and privacy over LTE.

\section{5G System Architecture}

The layered architecture of the 5G system is shown in Fig. 3. The bottom layer consists of the edge devices, wearables, and BANs, which are connected to each other via D2D (device-to-device) connections, and this system of small cell networks is further connected to the edge server/edge cloud via SBS (small base station) transmission. The network is of distributed type among the devices and edge server for this layer. The next layer is made

Table 3 Comparison of $5 \mathrm{G}$ with existing wireless technologies

\begin{tabular}{lllll}
\hline Technology & Speed & Power & Distance & Latency \\
\hline Sigfox & $450 \mathrm{kbps}$ & $0.2 \mathrm{w}$ & $20 \mathrm{miles}$ & $1-30 \mathrm{sec}$ \\
LoRA & $50 \mathrm{kbps}$ & $0.1 \mathrm{w}$ & $10 \mathrm{miles}$ & $1-10 \mathrm{sec}$ \\
Ingenu & $<1 \mathrm{mbps}$ & $0.1 \mathrm{w}$ & $50+\mathrm{miles}$ & $25+\mathrm{sec}$ \\
LTE-M & $1 \mathrm{mbps}$ & $0.5 \mathrm{w}$ & $6+\mathrm{miles}$ & $100 \mathrm{~ms}$ \\
NB-IoT & $250 \mathrm{kbps}$ & $0.5 \mathrm{w}$ & $6+\mathrm{miles}$ & $5 \mathrm{sec}$ \\
EC-GSM-IoT & $240 \mathrm{kbps}$ & $0.5 \mathrm{w}$ & $6+\mathrm{miles}$ & $2 \mathrm{sec}$ \\
Classic bluetooth & $1-3 \mathrm{mbps}$ & $1 \mathrm{w}$ & $100 \mathrm{~m}$ & $100 \mathrm{~ms}$ \\
BLE & $1-2 \mathrm{mbps}$ & $0.01-0.5 \mathrm{w}$ & $<100 \mathrm{~m}$ & $6 \mathrm{~ms}$ \\
Zigbee & $250 \mathrm{kbps}$ & $0.1 \mathrm{w}$ & $10-100 \mathrm{~m}$ & $>140 \mathrm{~ms}$ \\
WLAN & $100 \mathrm{mbps}$ & $100 \mathrm{w}$ & $50 \mathrm{~m}$ & $20-40 \mathrm{~ms}$ \\
$5 G$ & $10 \mathrm{gbps}$ & $1.4-2 \mathrm{kw}$ & $10 \mathrm{miles}$ & $>1 \mathrm{~ms}$ \\
\hline
\end{tabular}




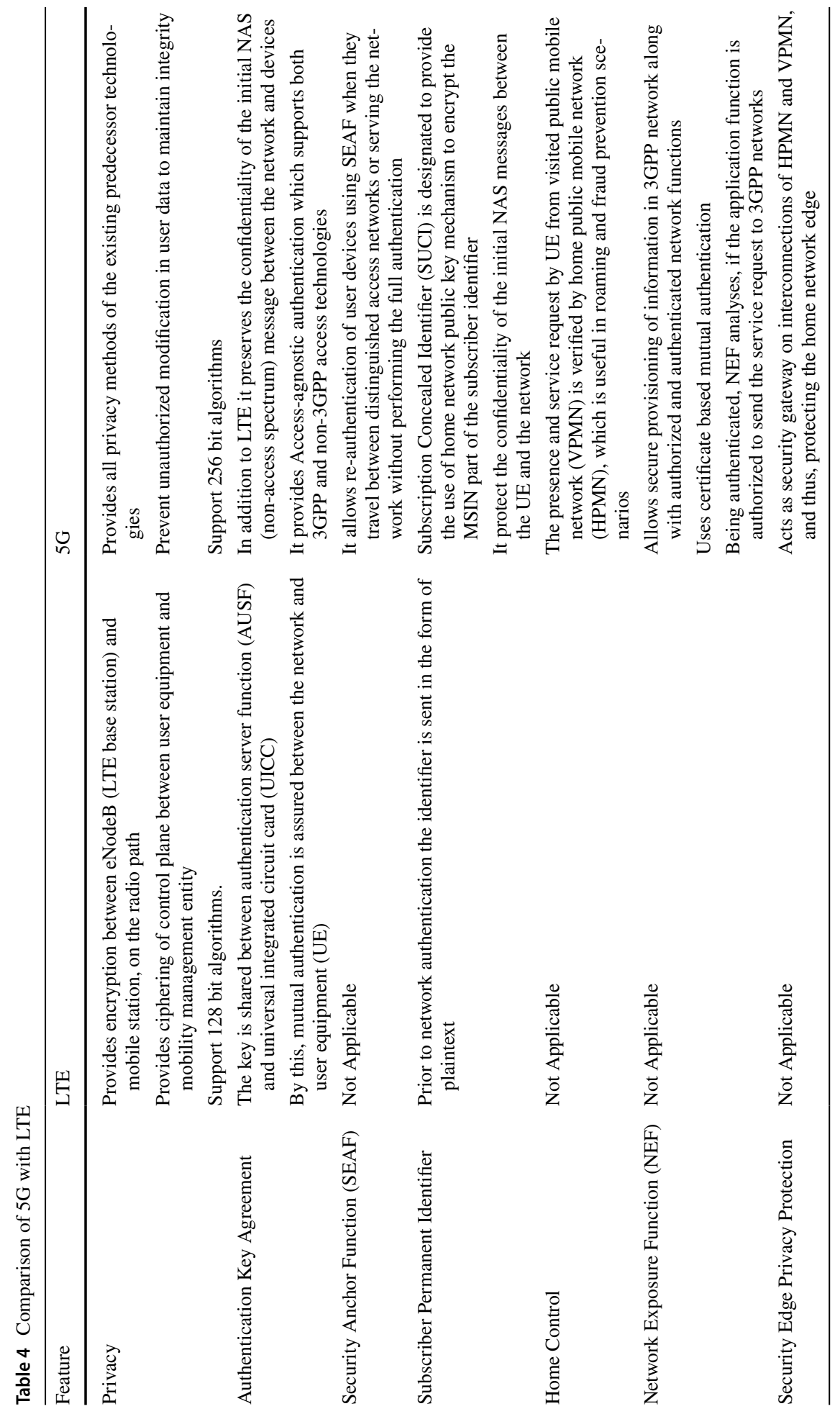




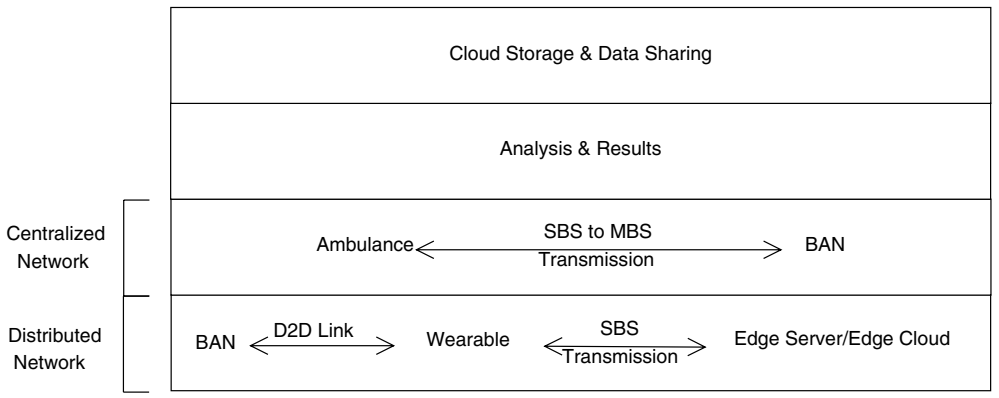

Fig. 3 5G system architecture

up of a Macro base station (MBS) type systems (connected ambulance, BAN). They are connected to each other by SBS to MBS transmission and network type for this layer is of a centralized network. The data generated by these layers are sent to the upper layer for analysis and result generation. The data is analyzed for finding the abnormality in gathered data, so the disease can be diagnosed based on gathered data and reports shared by patients. The generated results are then distributed to the family of the patient, hospital server, and the cloud storage for future reference.

\section{Applicability of 5G in loMT Applications}

\subsection{Remote Patient Monitoring}

Different types of sensors are placed or the concerned person is equipped with some wearables for accomplishing the remote monitoring task. Remote monitoring of any patient, specifically elderly people, deals with efficient and continuous monitoring of all the behavioral aspects of a person (heart rate or pulse rate, body temperature, breathing rate, oxygen level, respiration rate, blood pressure, etc.) for providing the better care and medications as and when required. It takes the record of glucose level, sugar level, electrocardiogram, any type of skin or eye disorder on a periodic basis. The report is generated after a selected period of time and sent to the doctor so that the doctor can find out if there is any problem on an a-priory basis.

\subsection{Connected Ambulance}

The ambulance is connected to the hospital server and the local server through which the patient is connected to the hospital or the doctor. These ambulances are equipped with first aid kit, along with sphygmomanometers, electrocardiogram, and ultrasound devices. Thus, the paramedical staff accompanying the patient can perform the required tests in the ambulance itself and send the report to the concerned doctors. It makes the doctor done with essential preparations before the patient arrives at the hospital and can serve the patient as soon as he/she comes. As $5 \mathrm{G}$ supports the mobility up to $100-150 \mathrm{~km} / \mathrm{h}$, thus, it supports road, railway, and air ambulances. 


\subsection{Virtual Consultation}

It is the process of consulting a doctor over an audio or video call, as per the requirements. The doctor talks or examine the person, as per the type of call, and then suggest the required medications on the call itself. Such type of consultation is very much useful in the current COVID-19 situation, and for other infectious diseases also. As, in the present COVID-19 situation, physical meetings are enhancing the rate of spreading highly, the performanceenhanced (low latency, high throughput, low packet loss, high data rate, etc.) 5G networks can help in reducing this spreading rate exponentially. Virtual consultation can reduce the travel cost along with the overcrowding of patients and their attendants at the hospital. Thus, the patient requires emergency handling can serve in a hassle-free environment.

\subsection{Real Time Maintenance}

It is the process of examining all the hardware devices and predicting their failure in advance. This can minimize the time taken in repairing or replacing that particular healthcare device, so continuous monitoring of the patient can happen.

\subsection{Augmented Reality (AR) and Virtual Reality (VR)}

AR and VR provide powerful and more interactive methods for exploring and interacting with medical data so that a natural and interactive environment can be provided to the doctors and patients virtually along with better realtime experience. It provides a simulated 3D digital experience to the user in a realistic way in the form of a lightweight stereoscopic head-mounted display, for providing the ehealthcare practitioner a seamless environment for interacting with the patient remotely [32]. The medical surgeon is assisted by the AR glasses at the time of various medical procedures by providing important pieces of information about the disease along with the surgical procedure. VR helps in training of healthcare personnel for performing the simulating surgeries. It requires a 360 video stream with 10 bit per pixel, and a data rate of at least $2.1 \mathrm{Gbps}$, which is possible by using $5 \mathrm{G}$.

\subsection{Robotic Surgery}

Robotic surgery is the process of performing and completing the surgical procedures with the help of advance and smart robots. In it, the surgeon instructs the remote robot to perform the surgical procedures in the at-most latency of $1 \mathrm{~ms}$. For example, the InMotion ARM, a product of bionik labs (https://www.bioniklabs.com/products/ inmotion-arm), helps the healthcare professionals to perform the biomedical surgeries remotely [33].

\subsection{Cloud Computing}

This part contributes to the registration, location, and other related data upload regarding the health center, patient data uploading, treatments and check-up phase, and the 
emergency phase. It establishes a mutually exclusive environment among patients, cloud servers, doctors, and healthcare centers, for preserving individual privacy and communication security. The cloud-based secure and efficient framework (CSEF) [34], establishes the session key among the pairs of doctor \& cloud, patient \& cloud, healthcare center \& patient, and healthcare center \& cloud, for the satisfaction of several security attributes.

Moreover, this section elaborates on several applications of the Internet of medical things and the applicability of $5 \mathrm{G}$ in those specific applications. Further, Table 5 provides a mapping between the required fields the applicability of $5 \mathrm{G}$ for the particular application. We use 1 (most required) and - (less attention can be tolerable) symbol in the table.

\section{5G NR Architecture}

5G system has been aimed to fulfill the current demands of smart ecosystem and highly mobile society. In near future, IoT applications come with diverse functionality and performance requirements, which will be supported by $5 \mathrm{G}$ networks. $5 \mathrm{G}$ aims to facilitate various key performance indicators of the upcoming new cases with cost efficient solution. Moreover, 5G systems provide a wide range of services to commercial enterprises, consumers, and third-party service providers.

In Fig. 4, we can see that existing architecture is made of an integrated part of control plane and UPF. In comparison of traditional architecture, control plane architectural components significantly simplifies deployment and development of the evolved high speed and reliable services with plug and play deployment, flexible orchestration architectural approach, which is a solid foundation for $5 \mathrm{G}$.

Control Plane is used for exchange of information between two neighborhood nodes to support the functions in the mobile telecommunications system, which is used to establish and maintain the user plane between two interconnected nodes but it doesn't facilitate end-to-end communication services. Additionally, the control plane is used to maintain the forwarding path to exchange the information for operation to the service of $5 \mathrm{G}$.

User Plane Function (UPF) is one of the fundamental parts of 5G architecture. UPF facilitates the data plane flow for Control and User Plane Separation (CUPS) strategy, which was firstly, introduced as an extension of Evolved Packet Cores (EPCs) in the release 14 of 3GPP. Additionally, the main goal of CUPS is to support 5G NR to facilitate the services of $5 \mathrm{G}$ for early IoT applications along with higher data rate. AMF is only responsible to handle all the connection and session related information from the UE including mobility management tasks.

\section{Results}

In this section, we consider a scenario of grid length $1000 \mathrm{~m}$ for simulation purpose. Further, we simulate over different frequency range FR1 \& FR2 and find the optimal throughput and latency for $5 \mathrm{G}$ NR. 


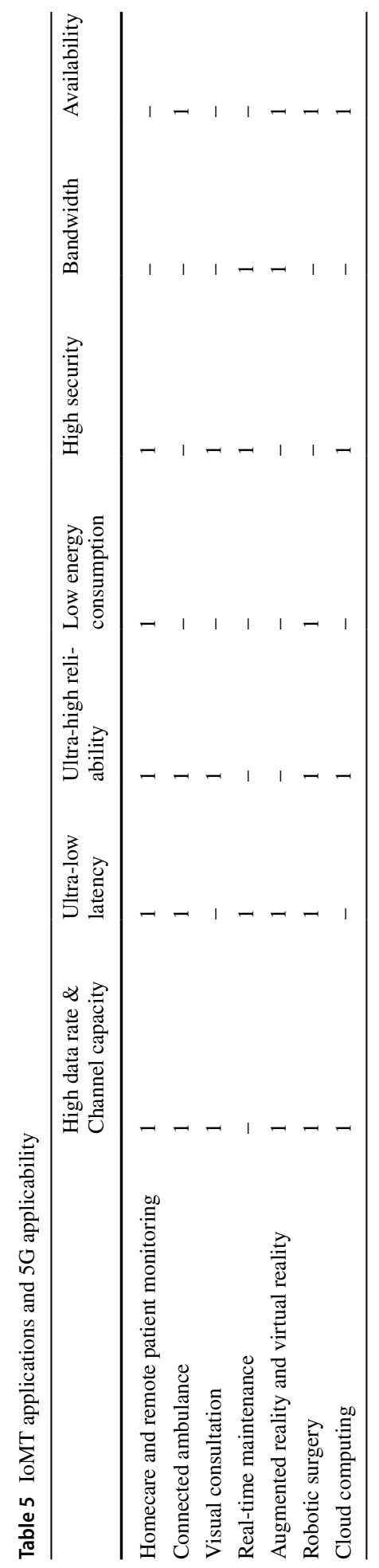




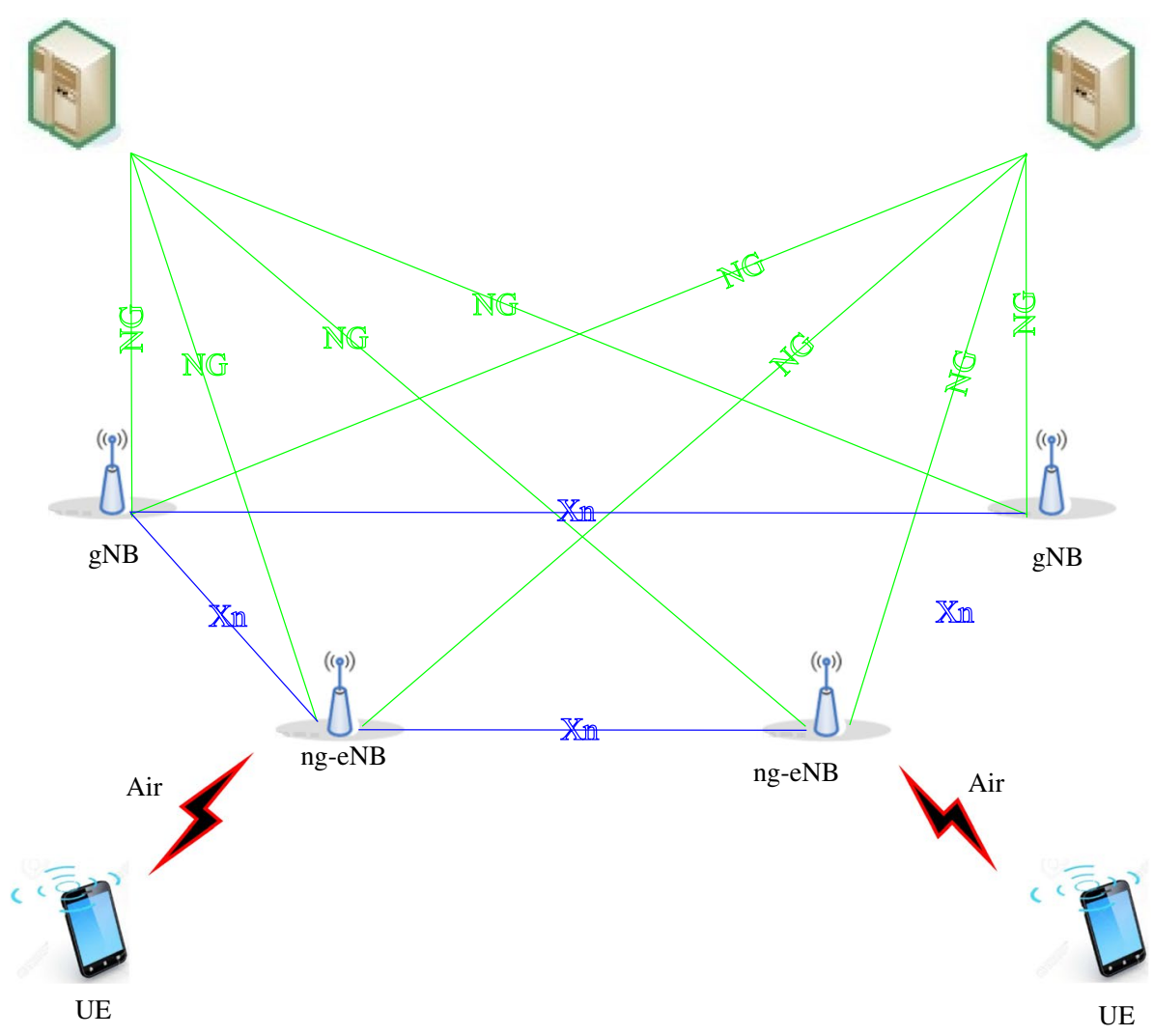

Fig. 4 Areal representation of 5G $600 \mathrm{MHz} \& 5 \mathrm{G}$ mm waves

\subsection{Simulation Setup for Performance Evaluation}

In this paper, the simulation to evaluate the performance is carried out in NetSim (Network Simulator and Emulator) on 64-bit Intel ®I7 2.30 GHz CPU with 12 GB RAM. NetSim is the industry's leading tool especially its functionality over 5G NR technology, which is widely accepted by more than 400+ reputed organizations. Moreover, to the best of our knowledge NetSim is one of the best tools available to evaluate the performance of communication technology over different related matrices. Further, it facilitates simulation over the discrete events with event-level debugging to inspect other aspects.

NetSim supports hardware as well as software control over the simulation environment. In our simulation, we have implemented 5G NR architecture over NetSim using UE, gNB, ng-eNB, NG, Xn components, which are available in NetSim.

Apart from the above functionalities, NetSim provides the privilege to access the physical layer, data link layer, and network layer for the industry and research community. Thus, it can set-up the environment for experimental purpose.

We know that, $5 \mathrm{G}$ is a next level technology but it is comparable with $4 \mathrm{G}$. The difference in the performance of $4 \mathrm{G}$ and $5 \mathrm{G}$ depends on the physical layer configuration 
of both technologies. Thus, we consider various numerologies for FR1 $(\mu=0,1,2)$ and FR2 $(\mu=2,3)$, which operate on both Time Division Duplex (TDD) and Frequency Division Duplex (FDD). However, in this work, we have considered FR1 only, because FR1 facilitates a comparable environment for 4G and 5G. Moreover, we have evaluated the performance over different bandwidth of the channel, which varies between 10 to $100 \mathrm{MHz}$. Finally, we have evaluated the performance of 4G and 5G and provide a comparative view.

In this work, we are evaluating the performance of $4 \mathrm{G}$ and $5 \mathrm{G}$. The main aim of both of the technologies is to achieve the highest throughput with low latency in a pervasive environment, which affects a number of noises that occur in a real-time environment. Thus, we have evaluated the throughput against signal-to-noise ratio and various career aggregation interfaces. Finally, we compare the average throughput and latency of $4 \mathrm{G}$ and $5 \mathrm{G}$ against a number of end-user devices.

Table 6 provides more details about the hardware and software configuration.

\subsection{Throughput Calculation}

Throughput is the measure of total amount of data that is transferred from one location (source) to the other location (destination) in a given time. We have used the following throughput formula for 5G NR specified by 3GPP technical specification 38.306 [35] for maximum throughput, which can be defined as:

$$
\operatorname{Throughput}(M b p s)=10^{-6} \cdot \sum_{j=1}^{J}\left[v_{\text {Layers }}^{(j)} \cdot Q_{m}^{(j)} \cdot f^{(j)} \cdot R_{\max } \cdot \frac{N_{P R B}^{B W(j), \mu} \cdot 12}{T_{s}^{\mu}} \cdot\left(1-O H^{(j)}\right)\right]
$$

Where, $\mathrm{J}$ denotes number of aggregated components carrier in bands. CA merge the multiple components carrier to achieve higher throughput, which automatically increases the overall system throughput. $v_{\text {Layers }}^{(j)}$ denote the maximum number of layers for $J$ th carrier components, which is depend in the radio channel. $Q_{m}^{(j)}$ is the maximum modulation order. $f^{(j)}$ is the scaling factor. $T_{s}^{\mu}$ denote the average duration of OFDM for the numerology $\mu$. $O H^{(j)}$ denote the overhead.

Table 6 Hardware and software configuration

\begin{tabular}{ll}
\hline Hardware and software & Specification \\
\hline Processor & Intel I7 $2.30 \mathrm{GHz}$ \\
Memory & $12 \mathrm{~GB}$ \\
Operating System & Windows 7 \\
Environment & $1000 \mathrm{~m}$ grid \\
Distance between $g_{N} B$ and UE & Varies between 100 and $1000 \mathrm{~m}$ \\
Packet size & 1460 Bytes \\
Packet arrival rate & 2000 to 20,000 micro second \\
\hline
\end{tabular}


Fig. 5 Throughput for FR1 and FR2 at various SNR

Fig. 6 Throughput for various numerologies
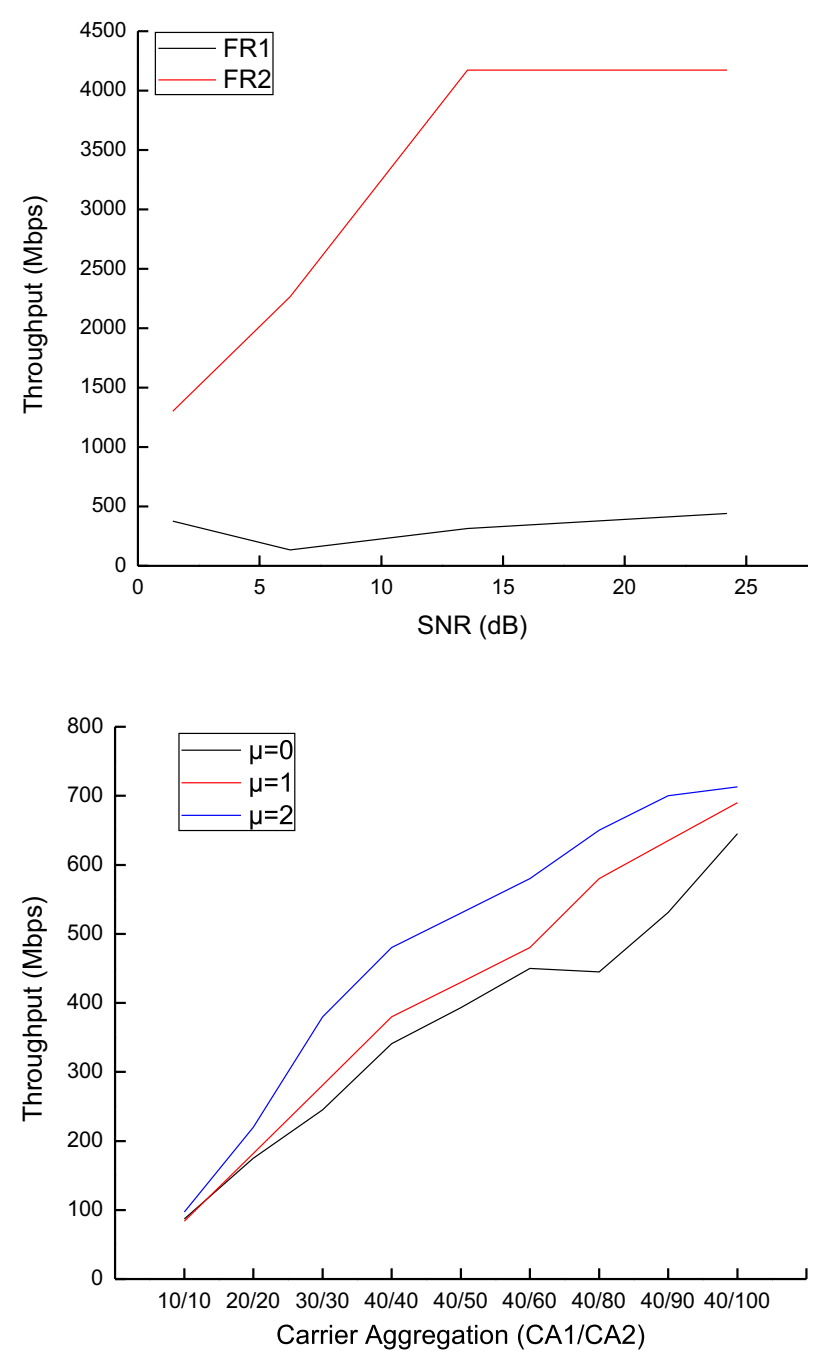

\subsection{FR1 and FR2}

In 5G NR there are two different frequency range are available FR1 and FR2. FR1 are much similar to traditional communication traffic, whereas FR2 aims to provide short range but very high data rate capability using $5 \mathrm{G}$ NR. To illustrate the difference, we have setup a $1000 \mathrm{~m}$ grid environment, in which we establish communication between gNB and UE.

To provide near real-time results, we choose to draw the throughput graph against SNR, which is affected by some other network factor like pathloss \& received power. In Fig. 5, we draw the graph between SNR and Throughput to illustrate the effect of SNR on the throughput. FR2 facilitates much higher throughput approximately 10 times as compared to FR1, but after a certain point, FR2 provides a nearly constant throughput against SNR. At the same time, FR1 could not provide that much higher throughput, because of the limited frequency range of less than $6 \mathrm{GHz}$ and extending up to $7.125 \mathrm{GHz}$, whereas FR2 supports the frequency range of 24.25 to $52.6 \mathrm{GHz}$. Additionally, FR2 is based on short-range 
communication with a higher data rate but FR1 which is molded towards LTE 4G could not provide that much high data rate. On the contrary, FR1 supports long-range communication as compared to FR2. Thus, we can say that $5 \mathrm{G}$ is more preferable choice as compared to $4 \mathrm{G}$ (Fig. 6).

\subsection{Throughput Against Various Numerologies}

We evaluate the throughput for various numerologies for $\mu=0,1,2$. Additionally, we choose two carrier aggregations (CA1/CA2) bandwidth. The value of CA1 bandwidth changes as 10, 20, 30, and $40 \mathrm{MHz}$ and CA2 bandwidth changes as 10, 20, 30, 40, 50, 60, 70, 80, 90, $100 \mathrm{MHz}$. Further, we measure the throughput for the above metrics. Fig. 7 illustrate the throughput for various numerologies. As the channel bandwidth increases it provide the higher throughput. Further, $\mu=2$ provides the better results as compare to $\mu=0$ and $\mu=1$, because it $120 \mathrm{Khz}$ where $\mu=0$ supports $15 \mathrm{Khz}$ and $\mu=1$ supports $30 \mathrm{Khz}$. Thus, $\mu=2$ provide the higher throughput.

\subsection{Latency Against Various Numerologies}

In the Fig. 7, we can see that $\mu=2$ provide the lowest latency for the various bandwidth as compare to $\mu=0$ and $\mu=1$. The possible reason of that can be find in frame structure of the numerology $\mu=2$. $\mu=2$ supports 40 frames and 4 subframes. So, it can process a unit amount of data in 125 microseconds whereas $\mu=0, \mu=1$ process that unit amount of data in 250 microsecond and 500 microsecond. Thus, $\mu=2$ provide much lesser latency as compared to other.

\subsection{G vs 5G: Performance Comparison}

In this section, we provide a comparative analysis between $4 \mathrm{G}$ LTE and $5 \mathrm{G} \mathrm{NR}$. For the $4 \mathrm{G}$ configuration, we use FR $1, \mu=0$ with the bandwidth of $20 \mathrm{Mhz}$, which represents $4 \mathrm{G}$ configuration. In comparison of this $4 \mathrm{G}$ scenario, we use FR2, $\mu=3$ with the

Fig. 7 Latency for various numerologies

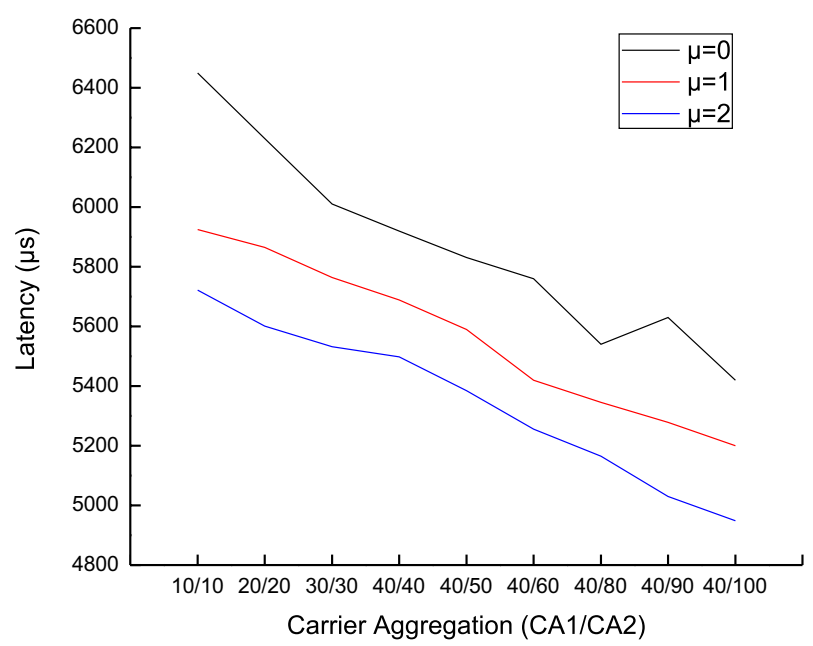


Fig. 8 Aggregated throughput of $4 \mathrm{G}$ and $5 \mathrm{G}$ for increasing number of devices

Fig. 9 Average delay of $4 \mathrm{G}$ and $5 \mathrm{G}$ for increasing number of devices
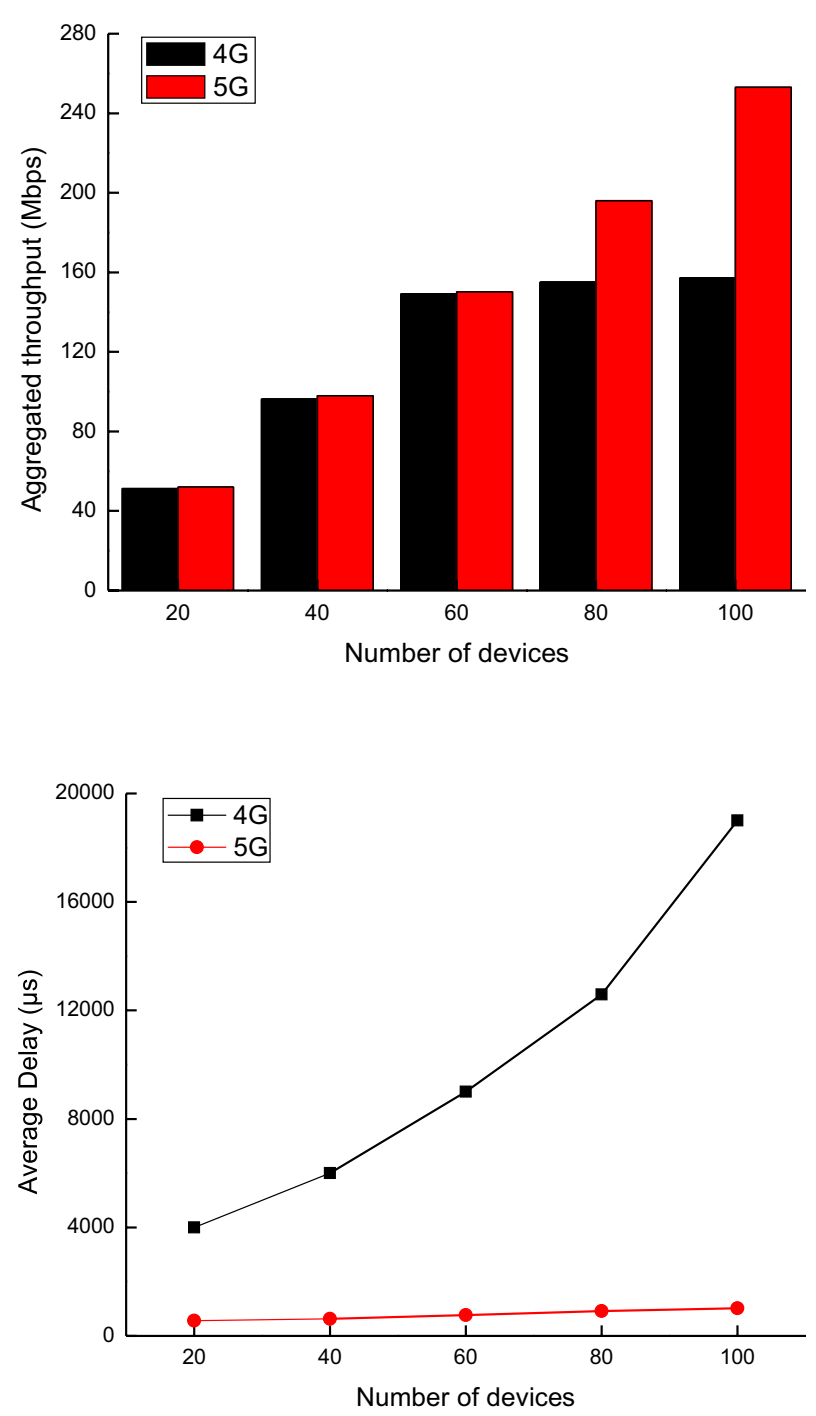

bandwidth of $100 \mathrm{MHz}$, which represent the $5 \mathrm{G}$ configuration. Further, from IoT application point of view, we use one source station and number of user equipments changes to $20,40,60,80,100$, which are used to download the data from the source station. Further, we measure the throughput and delay for the above IoT application

From the Fig. 8, we can see that initially we got almost equal throughput but as the number of user equipments increases the throughput start decreasing in 4G. However, $5 \mathrm{G}$ achieve the necessary bandwidth for each user because of the higher available bandwidth in it.

From Fig. 9 we can see that in 5G latency increases linearly whereas in $4 \mathrm{G}$ the latency increases exponentially. The possible reason for this behavior is the sufficient advancements in the 5G NR communication infrastructure. On the basis of the results, we can conclude that $5 \mathrm{G}$ NR is the most suitable solution for IoT applications. 


\section{Conclusion and Future Scope}

In this paper, we illustrate the working principle of $5 \mathrm{G}$, the need for $5 \mathrm{G}$ in the healthcare domain, and healthcare applications where the real-time solution is needed. We provide the $5 \mathrm{G}$ taxonomy in terms of $5 \mathrm{G}$ objectives, requirements of $5 \mathrm{G}$ for fulfilling these $5 \mathrm{G}$ objectives, the approaches need to be opted for accomplishing the $5 \mathrm{G}$ objectives. Further, we discuss the short-range and longrange $5 \mathrm{G}$ communication technologies along with the $5 \mathrm{G}$ performance metrics. Moreover, we proposed two distinct architectures of $5 \mathrm{G}$ NR. First is the fourlayer $5 \mathrm{G}$ architecture with network slicing. The second is $5 \mathrm{G}$ NR architecture, which provides a better understanding of the functioning of the control plane and user plane along with the concept of control and user plane separation (CUPS).

The result section provides distinct values of throughput for different frequency range FR1 \& FR2, for 5G NR. Further, we provide varying values of throughput and latency for various numerologies $(\mu=0,1,2)$ with CA1 and CA2. Moreover, we administer a comparative analysis for $4 \mathrm{G}$ LTE \& $5 \mathrm{G}$ NR and infer that $5 \mathrm{G}$ NR can accommodate a much higher number of devices than $4 \mathrm{G}$ and $5 \mathrm{G}$ NR facilitates 10 times lower latency in comparison with $4 \mathrm{G}$.

The comparison of LTE and $5 \mathrm{G}$ justifies that $5 \mathrm{G}$ provides a more secure environment for communication and more data privacy than LTE. The high speed and ultra-low latency features of $5 \mathrm{G}$ make it highly applicable for timesensitive applications, like healthcare, where a delay of even a second can cause a person's death or reach the severity of the disease to the worst extreme. In the near future, we will try to implement the 5G NR in various IoT applications, like environment monitoring, cloud data processing, etc. in a more realistic manner. Further, in this paper, we analyzed the performance against throughput $\&$ latency, in future, we will work on analyzing the performance of other 5G NR features, like, response time, connection density, communication availability, user mobility support.

\section{References}

1. Alwan, A. (2011). Global status report on noncommunicable diseases 2010. World Health Organization.

2. Rashid, S. \& Razak, S. A. (2019). Big data challenges in 5G networks. In 2019 Eleventh international conference on ubiquitous and future networks (ICUFN), Zagreb, Croatia, pp. 152-157. https://doi.org/ 10.1109/ICUFN.2019.8806076.

3. Latif, S., Qadir, J., Farooq, S., \& Imran, M. A. (2017). How 5g wireless (and concomitant technologies) will revolutionize healthcare? Future Internet, 9(4), 93.

4. Cisotto, G., Casarin, E., \& Tomasin, S. (2020). Requirements and enablers of advanced healthcare services over future cellular systems. IEEE Communications Magazine, 58(3), 76-81.

5. World Health Organization Global Health and Aging. (2011). World Health Organization, Geneva, Switzerland.

6. Soldani, D., Fadini, F., Rasanen, H., Duran, J., Niemela, T., Chandramouli, D., \& Nanavaty, N. (2017). 5G mobile systems for healthcare. In 2017 IEEE 85th vehicular technology conference (VTC Spring), (pp. 1-5). IEEE.

7. Rehman, I. U., Nasralla, M. M., Ali, A., \& Philip, N. (2018). Small cell-based ambulance scenario for medical video streaming: A 5G-health use case. In 2018 15th International conference on smart cities: Improving quality of life using ICT \& IoT (HONET-ICT) (pp. 29-32). IEEE.

8. Yao, M., Sohul, M., Marojevic, V., \& Reed, J. H. (2019). Artificial intelligence defined 5G radio access networks. IEEE Communications Magazine, 57(3), 14-20. https://doi.org/10.1109/MCOM.2019. 1800629. 
9. Ahad, A., Tahir, M., \& Yau, K. L. A. (2019). 5G-based smart healthcare network: Architecture, taxonomy, challenges and future research directions. IEEE Access, 7, 100747-100762.

10. Nasimi, M., Habibi, M. A., Han, B., \& Schotten, H. D. (2018). Edge-assisted congestion control mechanism for $5 \mathrm{G}$ network using software-defined networking. In 2018 15th International symposium on wireless communication systems (ISWCS) (pp. 1-5). IEEE.

11. Chowdhury, M. Z., Hossan, M. T., Shahjalal, M., Hasan, M. K., \& Jang, Y. M. (2020). A new 5G eHealth architecture based on optical camera communication: An overview, prospects, and applications. IEEE Consumer Electronics Magazine.

12. Wang, C. X., Di Renzo, M., Stanczak, S., Wang, S., \& Larsson, E. G. (2020). Artificial intelligence enabled wireless networking for $5 \mathrm{G}$ and beyond: Recent advances and future challenges. IEEE Wireless Communications, 27(1), 16-23.

13. Ahmed, I., Karvonen, H., Kumpuniemi, T., \& Katz, M. (2020). Wireless communications for the hospital of the future: Requirements, challenges and solutions. International Journal of Wireless Information Networks, 27(1), 4-17.

14. Liu, E., Effiok, E., \& Hitchcock, J. (2020). Survey on health care applications in 5 G networks. IET Communications, 14(7), 1073-1080.

15. Annapoorani, S., Somakumar, R., Devi, P. V., Emimal, L., \& Selvan, B. B. Smart wearable sensor for health monitoring of elderly people.

16. Dzogovic, B., Santos, B., Jacot, N., Feng, B., \& Van Do, T. (2020, May). Secure healthcare: 5G-enabled network slicing for elderly care. In 2020 5th International conference on computer and communication systems (ICCCS) (pp. 864-868). IEEE.

17. Ioannou, I., Vassiliou, V., Christophorou, C., \& Pitsillides, A. (2020). Distributed artificial intelligence solution for D2D communication in 5G networks. IEEE Systems Journal, 14(3), 4232-4241. https://doi.org/10.1109/JSYST.2020.2979044.

18. Chen, M., Yang, J., Hao, Y., Mao, S., \& Hwang, K. (2017). A 5G cognitive system for healthcare. Big Data and Cognitive Computing, 1(1), 2.

19. Hewa, T., Braeken, A., Ylianttila, M., \& Liyanage, M. Multi-access edge computing and blockchain-based secure telehealth system connected with $5 \mathrm{G}$ and IoT.

20. Ning, Z., Dong, P., Wang, X., Hu, X., Guo, L., Hu, B., \& Kwok, R. Y. (2020). Mobile edge computing enabled $5 \mathrm{G}$ health monitoring for Internet of medical things: A decentralized game theoretic approach. IEEE Journal of Selected Areas Communications, 1-16.

21. Liu, G., \& Jiang, D. (2016). 5G: Vision and requirements for mobile communication system towards year 2020. Chinese Journal of Engineering, 2016, 8.

22. AlQahtani, S. A. (2020). An efficient resource allocation to improve QoS of $5 \mathrm{G}$ slicing networks using general processor sharing-based scheduling algorithm. International Journal of Communication Systems, 33(4), e4250.

23. Moysen, J., \& Giupponi, L. (2018). From 4G to 5G: Self-organized network management meets machine learning. Computer Communications, 129, 248-268.

24. Alnahdi, A. \& Liu, S. (2017). Mobile Internet of Things (MIoT) and its applications for smart environments: A positional overview. In 2017 IEEE International Congress on Internet of Things (ICIOT), Honolulu, HI, pp. 151-154. https://doi.org/10.1109/IEEE.ICIOT.2017.26.

25. Parvez, I., Rahmati, A., Guvenc, I., Sarwat, A. I., \& Dai, H. (2018). A survey on low latency towards 5G: RAN, core network and caching solutions. IEEE Communications Surveys \& Tutorials, 20(4), 3098-3130.

26. Rashid, A., Tripathi, Y., Prakash, A., \& Tripathi, R. (2019). Load aware energy-balanced data gathering approach in CRSNs. IET Wireless Sensor Systems, 9(3), 143-150.

27. Rao, S. K., \& Prasad, R. (2018). Impact of 5G technologies on industry 4.0. Wireless Personal Communications, 100(1), 145-159.

28. Yan, M., Feng, G., Zhou, J., Sun, Y., \& Liang, Y. C. (2019). Intelligent resource scheduling for 5G radio access network slicing. IEEE Transactions on Vehicular Technology, 68(8), 7691-7703.

29. Khan, Z., Fan, P., Abbas, F., Chen, H., \& Fang, S. (2019). Two-level cluster based routing scheme for 5G V2X communication. IEEE Access, 7, 16194-16205.

30. Najm, I. A., Hamoud, A. K., Lloret, J., \& Bosch, I. (2019). Machine learning prediction approach to enhance congestion control in 5G IoT environment. Electronics, 8(6), 607.

31. Vidakis, K., Mavrogiorgou, A., Kiourtis, A., \& Kyriazis, D. (2020). A comparative study of shortrange wireless communication technologies for health information exchange. In 2020 International conference on electrical, communication, and computer engineering (ICECCE), Istanbul, Turkey, pp. 1-6. https://doi.org/10.1109/ICECCE49384.2020.9179478. 
32. Desselle, M. R., Brown, R. A., James, A. R., Midwinter, M. J., Powell, S. K., \& Woodruff, M. A. (2020). Augmented and virtual reality in surgery. Computing in Science \& Engineering, 22(3), 18-26. https://doi.org/10.1109/MCSE.2020.2972822.

33. Kamruzzaman, M. M. (2020). Architecture of smart health care system using artificial intelligence. In 2020 IEEE international conference on multimedia \& expo workshops (ICMEW) (pp. 1-6). IEEE.

34. Kumari, A., Kumar, V., Abbasi, M. Y., Kumari, S., Chaudhary, P., \& Chen, C. M. (2020). Csef: Cloud-based secure and efficient framework for smart medical system using ecc. IEEE Access, 8 , $107838-107852$.

35. 3GPP 5G (2018). 5G NR User Equipment (UE) radio access capabilities. 3GPP Technical Specification 38.306.

36. Cui, T. J., Zoha, A., Li, L., Shah, S. A., Alomainy, A., Imran, M. A., \& Abbasi, Q. H. (2020). Revolutionizing future healthcare using wireless on the walls (WoW). arXiv preprint arXiv:2006.06479.

Publisher's Note Springer Nature remains neutral with regard to jurisdictional claims in published maps and institutional affiliations.

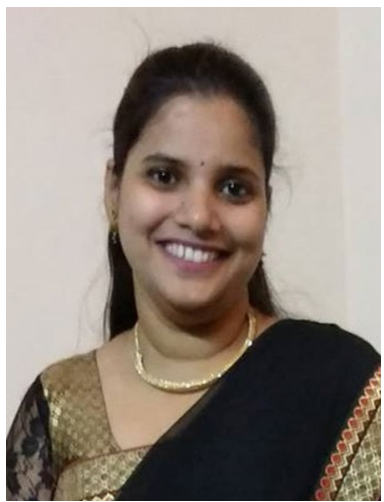

Lalita Mishra received the B.Tech. degree in Computer Science and Engineering from Uttar Pradesh Technical University, Lucknow, India, in 2011 and M. Tech. from International Institute of Information Technology, Bhubaneswar. Currently, she is working towards $\mathrm{Ph} . \mathrm{D}$ at Indian Institute of Information Technology, Allahabad in the department of Information Technology. Her research interest include Internet of Things, Cloud Computing, Wireless Sensor Networks, Edge Computing, and $5 \mathrm{G}$.

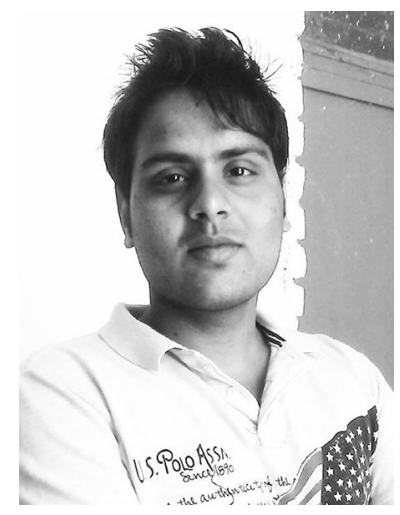

Vikash is working as an Assistant Professor in the Department of Computer Science \& Engineering and Information Technology at Jaypee Institute of Information Technology, Noida, Uttar Pradesh. Prior to that, Vikash has been worked as Teaching Fellow in School of Computing Science and Engineering at VIT Bhopal University. Vikash has pursued $\mathrm{PhD}$ in research area of Wireless Sensor Networks, Internet of Things from Department of Information Technology at Indian Institute of Information Technology, Allahabad, India in 2021. He has completed his Master of Technology degree in Computer Science and Engineering from Kamla Nehru Institute of Technology, Sultanpur, India in 2015. Before that, he obtains a Bachelor of Technology degree in Computer Science and Engineering from Uttar Pradesh Technical University, Lucknow, India in 2013. His general research interest includes Internet of Things, Wireless Sensor Networks, Wireless Networks, AI enabled Internet of Things and Pervasive Computing. 


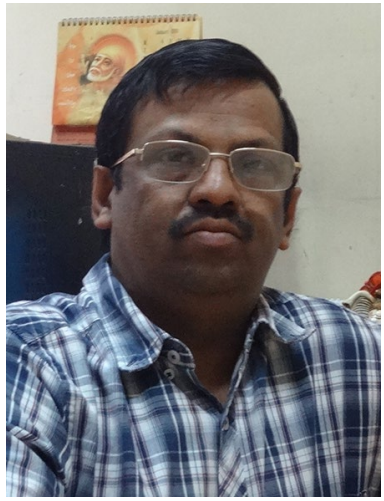

Shirshu Varma after completing Ph.D. served many reputed organizations like, BIT Mesra Ranchi, CDAC Noida in the capacity of lecturer, Sr. Lecturer and Principal project engineer. Presently working as a Professor in Indian Institute of Information Technology-Allahabad (IIITAllahabad). He has about 25 years of experience of teaching and research. He has published about 50 papers in international and national journals and conferences of repute and is the author of 04 book chapters. Number of citations of his papers is approximately 60 . His area of work includes: Wireless sensor networks- coverage amd connectivity, Sensor deployment and localization, Wireless sensor statistical routing etc., WI-FI, WiMA. 